Colás Bravo, Pilar; Contreras Rosado, José Antonio (2013). La participación de las familias en los centros dē Educación Primaria. Revista de Investigación Educativa, 31 (2), 485-499.

http://dx.doi.org/10.6018/rie.31.2.171031

\title{
LA PARTICIPACIÓN DE LAS FAMILIAS EN LOS CENTROS DE EDUCACIÓN PRIMARIA*
}

\author{
Pilar Colás Bravo y José Antonio Contreras Rosado \\ Universidad de Sevilla
}

\section{RESUMEN}

El objetivo de esta investigación es conocer la respuesta de los padres a la oferta de participación que realizan los centros de Educación Primaria, así como los motivos y concepciones que promueven la colaboración de las familias con la oferta que reciben. Los modelos teóricos de Epstein y Vogels sirven de fundamento teórico y metodológico para el desarrollo de este estudio. La muestra asciende a 110 familias de alumnos de primaria y representa a una población 41.799 familias sevillanas. Para la recogida de datos se elabora un cuestionario ad hoc basado en la conjunción de los modelos teóricos de Vogels y Epstein. Los resultados muestran las preferencias por unas determinadas opciones de colaboración, así como la coexistencia de concepciones y motivaciones que representan distintos niveles de implicación y compromiso con la educación.

Palabras clave: participación familiar, modelos, centros educativos, educación primaria.

\section{FAMILY INVOLVEMENT IN PRIMARY EDUCATION}

\author{
ABSTRACT \\ The aim of this research study was to analyse parents' involvement in primary schools \\ and the reasons and conceptions that may foster such involvement. The models of Epstein and \\ Correspondencia: \\ Pilar Colás Bravo. Departamento de Métodos de Investigación y Diagnóstico en Educación. Facultad de \\ Ciencias de la Educación. Universidad de Sevilla. C/ Pirotecnia, s/n. 41013 Sevilla. \\ E-mail de los autores: \\ Pilar Colás Bravo (pcolas@us.es) y José Antonio Contreras Rosado (josconros@alum.us.es) \\ * Investigación antecedente al proyecto: Students guidance at university for inclusion (Ref: 526600-LLP- \\ 1-2012-IT-Erasmus-Esin) Entidad financiadora: Lifelong learning programme. Call for proposals: EAC/27/11. \\ Erasmus programme. Erasmus multilateral projects.
}


Vogels served as a theoretical and methodological basis in this study. The sample comprised 110 families with primary school pupils. The population was made up of 41,799 families from Seville, Spain. The confidence level was set at 95\% and sampling error was \pm 10 . An ad hoc questionnaire based on the theoretical models of Epstein and Vogels was developed for data collection. The results showed that parents preferred certain collaboration options. Some conceptions and motivations combined, thus showing different levels of involvement in and commitment to education.

Keywords: family involvement, models, schools, primary school.

\section{INTRODUCCIÓN}

La participación de los padres en los centros educativos es uno de los indicadores clave de la Calidad de los Sistemas Educativos. De ahí que la colaboración de las familias con éstos en la formación de sus hijos sea una temática que ocupe la atención en dos planos; político-legislativo y científico.

En el año 2000, la Comisión Europea publica el Informe Europeo sobre Calidad de la Educación Escolar en el que establece dieciséis indicadores que marcan la categoría de la enseñanza. Uno de ellos es la cooperación de los padres, entendiendo que son agentes que pueden realizar contribuciones eficaces en el rendimiento de los alumnos y en la democratización de las escuelas.

En este mismo sentido el Programa de Formación de Padres de la Asociación Europea de Padres de Alumnos (EPA) constituye un ejemplo de cómo mejorar la calidad por medio de la cooperación y el diálogo escolar constructivo entre los padres y los profesores. Estos cursos de formación pretenden poner de relieve la importancia del papel de los padres y su responsabilidad en la educación de sus hijos.

A nivel nacional la participación de los padres en las instituciones educativas se recoge en la legislación española, de forma continuada en el tiempo. La primera referencia la tenemos en la Constitución de 1978 en su artículo 27 que señala dos niveles de participación de la comunidad educativa y, por tanto, de los padres: en la programación general de la enseñanza (apartado 5) y en el control y gestión de los centros financiados con fondos públicos (apartado 7).

Posteriormente sucesivas leyes orgánicas educativas (LODE de 1985, LOGSE de 1990 y LOPEG de 1995) desarrollan la participación, hasta que la Ley Orgánica de Calidad de la Educación (LOCE) de 2002 reforma la situación haciendo referencia a los "órganos de participación en el control y gestión de los centros" (Sección $3^{\underline{a}}$ ), donde aparece el Consejo Escolar en su composición.

Finalmente la actual Ley de Educación (Ley Orgánica de Educación LOE 2/2006) incide en la importancia de la cooperación de las familias con el centro educativo. En concreto, en su artículo 118 destaca que las Administraciones Educativas fomentarán y adoptarán medidas para potenciar la colaboración entre la familia y la escuela.

También en el actual borrador de Ley Orgánica para la Mejora de la Calidad Educativa (LOMCE) disponible en la página del Ministerio de Educación Cultura y Deporte (LOMCE versión 3 26/02/2013) plantea la participación de los padres en el Artículo 119 en relación con el funcionamiento y el gobierno de los centros educativos. 
Por tanto, esta temática se revela como prescriptiva en el funcionamiento de las escuelas, incidiéndose en una perspectiva de gestión. Sin embargo nos genera varios interrogantes ¿Cómo se concreta esta cooperación? ¿Solamente a nivel de organización y gestión? o ¿Qué tipo de colaboración educativa proponen las escuelas a las familias? Y ¿Cuál es el nivel de respuesta que reciben? De ahí que en este estudio pretendamos indagar sobre la respuesta que dan las familias a la oferta que hacen los colegios. El siguiente apartado presenta los resultados del rastreo bibliográfico sobre la investigación realizada en torno a la participación de los padres en los centros educativos.

\section{REVISIÓN DEL ESTADO DE LA CUESTIÓN}

\section{I. Estudios empíricos}

A nivel científico se identifican dos líneas de investigación claramente delimitadas. Una centrada en la identificación de niveles o grados de participación de los padres en las escuelas, indagando sobre diferentes ámbitos de colaboración y otra en los efectos o beneficios que conlleva la cooperación de las familias en las escuelas.

En la primera línea podemos citar la investigación de Santos Guerra (1997), en la que se detecta una escasa afluencia de votantes en elecciones al Consejo Escolar, 85 familias sobre un total de 1.675 y 52 sobre 1.521. Muy parecidas son las cifras que aporta Martín-Moreno (2000) sobre la participación de las familias en las mismas elecciones en centros de Educación Primaria (curso1995/1996) que registran índices de participación del $29.29 \%$ en centros públicos y del $47.10 \%$ en privados. A nivel autonómico Garreta (2008) estudia la tendencia asociativa en Andalucía, obteniendo una media de inscripción de las familias en las asociaciones de padres y madres del $48.22 \%$, además de valorar de forma insatisfactoria $(58.4 \%)$ su colaboración en las escuelas.

Más recientemente, el Consejo Escolar del Estado en el informe de 2012, con señala que la participación de las familias en las AMPAS (asociaciones de madres y padres del alumnado) oscila en torno al $60 \%$ en las etapas de Infantil y Primaria, bajando hasta un $50 \%$ en la Secundaria.

Otras aportaciones actuales (Lozano, Alcaraz y Colás, 2013) indagan sobre un total de 1.932 respuestas de familias autóctonas de la región de Murcia y emigrantes sobre las propuestas de colaboración que ofertan los centros educativos a los padres (AMPAS, Consejos Escolares, actividades escolares y extraescolares, etc.). Destacando que no se registran índices de participación familiar acordes con su relevancia, ya que la mayoría de las familias opinan que participan «algo» $(45,6 \%)$ o «poco» $(30,4 \%)$, cuando el centro docente en el que están escolarizados sus hijos convoca reuniones informativas sobre aspectos educativos.

Así pues los resultados obtenidos a lo largo de tres décadas, muestran una tendencia positiva. Sin embargo todavía no se alcanzan niveles óptimos deseables, dado su valor e importancia educativa que tiene, si consideramos los beneficios que aporta en cuanto a cohesión social, integración, y rendimiento escolar, entre otros.

La participación de las familias es un factor importante desde el punto de vista de la integración y cohesión social (Santos, Lorenzo y Priegue, 2011). Otros trabajos como el de Lozano, Alcaraz y Colás (2013) revelan que la participación de las familias en 
la escuela es un elemento clave en la integración de los niños y especialmente en los casos de niños inmigrantes.

Por otra parte tanto investigaciones nacionales como internacionales (Dearing, Kreider y Weiss 2006, Sanders 2005, Driessen, Smit y Sleegers, 2007, Ruiz de Miguel 2009) muestran la conexión entre participación familiar y rendimiento escolar, señalándose como un factor clave en el éxito académico. Estos estudios comprueban que el compromiso, tanto de profesores como de los padres, favorece los logros de los alumnos en matemáticas. Incluso en culturas orientales como el estudio de Chiu y Xihua (2008) se obtienen resultados similares llegando a concluir que la participación es uno de los aspectos más importantes para un alto rendimiento en esta disciplina. También coinciden con estos resultados el estudio de Barton (2001) en el que se concluye que la implicación de las madres del alumnado en la asignatura de ciencias es uno de los factores más influyentes en la consecución de altos resultados. También parece ser un aspecto importante en la promoción de la lectura, como lo demuestra el trabajo de Villiger, Niggli y Wandeler (2011).

En este sentido, el último informe publicado por la OECD (2012) muestra estimaciones, a través de modelos de regresión, del peso de la participación familiar en el rendimiento lector de los escolares. Esta se concreta en cuatro vertientes: a) vigilancia de los padres del progreso académico de sus hijos, así como de sus conductas, b) realización de actividades de voluntariado en el centro, c) ayuda en las tareas escolares en casa y d) intercambio de información con los tutores. Los resultados indican que todas estas formas de participación de los padres obtienen coeficientes significativos con relación al rendimiento en lectura.

Otros estudios (Córdoba, García, Luego, Vizuete y Feu, 2011; Fraguela, Lorenzo y Varela, 2011; Fan, Williams y Wolters, 2011) confirman que a mayor participación familiar, mayor rendimiento y motivación. La motivación, por tanto, parece ser otra parcela que se ha abordado desde la perspectiva de la participación de los padres. Jiménez (2006) evidencia la influencia que ejercen las familias sobre la educación de sus hijos/ as que afecta, no sólo a la motivación o a los resultados, sino al interés e implicación del alumnado por su propio desarrollo o educación. Aumenta, también, la satisfacción de las relaciones entre educadores y padres, mejorando tanto la motivación como las expectativas de ambos con respecto al proceso educativo (Sarramona y Roca, 2007).

También parece observarse una relación estrecha entre la implicación de las familias en la vida escolar de sus hijos/as y la participación en otros ámbitos de la vida de éstos, tal como indican Pomerantz, Moorman y Litwack (2007). Estos autores mantienen que los padres y madres que más se involucran en el ámbito educativo de sus hijos/as, igualmente se implican en otros ámbitos. Por lo que, al aumentar la participación en un campo concreto, en este caso el educativo, se contribuye a elevar esa implicación en otros terrenos que influyen en la vida del alumnado.

La exposición hasta aquí realizada concierne al valor educativo de la participación de los padres en los centros escolares, sin embargo otras facetas necesitan de un mayor desarrollo científico. Es preciso conocer de forma más precisa los motivos que regulan la mayor o menor implicación de las familias en la oferta que los centros realizan, así como los modelos de participación implícitos en la propuesta que los centros formalizan. En estas dos cuestiones se centrará nuestra aportación. 


\subsection{Modelos teóricos sobre participación}

La revisión de la bibliografía científica sobre esta temática, formalizada a través de bases de datos internacionales y nacionales, nos lleva a identificar dos modelos teóricos que permiten sistematizar, tanto los tipos de participación que pueden darse en los centros educativos de educación primaria (Epstein y otros 2002), como las concepciones que tienen los padres de la escuela, que podrían explicar el tipo de participación que adoptan (modelo de Vogels 2002). El modelo de Vogels, sistematiza las concepciones educativas de los padres en: consumidores, clientes, participantes y socios. Estos posicionamientos, conllevan diferentes niveles o grados de implicación familiar con el centro.

Así el término de "consumidores" concibe la educación como un producto que se consume y del que, si no se está satisfecho, se opta por la elección de otro que se considere mejor. La interiorización de este planteamiento se manifiesta cuando la participación de la familia se reduce a la mera elección del colegio que se piensa más adecuado para su hijo/a.

El término "clientes" se aplica cuando los padres asumen que los docentes son los expertos y responsables de la educación. Por lo tanto, los asuntos de la escuela recaerán siempre en ellos, ya que se entiende que los profesionales son los educadores.

La modalidad de "participantes" se refiere a que las familias se implican en la educación de sus hijos/as, colaborando activamente con los profesionales. Es propio de este modelo que las familias tengan iniciativas y planteen propuestas.

La denominación de "socios", conlleva el grado máximo de implicación de las familias ya que estas consideran que la educación de sus hijos debe afrontarse de forma colaborativa con el profesorado.

El modelo anterior pone el acento en las distintas concepciones que tienen las familias que inciden y determinan su participación en el centro. Sin embargo también es necesario tener presente la oferta de participación que el centro brinda a los padres. El modelo Epstein y otros (2002), nos permite identificar y clasificar el tipo de oferta de participación que hacen los centros a las familias. En él se identifican seis opciones: Ayuda a los padres (Parenting), Comunicación (Communicating), Voluntariado (Volunteering), Aprendizaje en casa (Learning at home), Toma de decisiones (Decision making), y Colaboración con el entorno (Collaborating with community).

La opción de Ayuda a los padres (Parenting), se centra en el apoyo del centro a los padres en lo relacionado con la crianza de los niños. La segunda iniciativa de Comunicación (Communicating), se basa en potenciar la comunicación mutua entre los docentes y las familias. El papel de los padres se circunscribe a recibir esa información y responder.

La tercera modalidad, Voluntariado (Volunteering), pretende fomentar el voluntariado de las familias en las actividades que realiza la escuela ya sean escolares, extraescolares o de cualquier tipo. La colaboración de las familias es desinteresada.

El cuarto tipo, Aprendizaje en casa (Learning at home), se orienta a desarrollar el aprendizaje en el hogar, bajo las directrices de la escuela. Las familias seguirán las sugerencias del colegio y el hogar será, en lo referente al proceso educativo, una continuación de la escuela.

La opción de Toma de decisiones (Decision making) se focaliza en la implicación de las familias y el alumnado en los órganos de gobierno y gestión del centro educativo. 
La función de las familias será motivar a sus hijos/as a formar parte del Consejo escolar del centro o de alguna asociación estudiantil, dando ejemplo ellos mismos con su participación en órganos colegiados o en la asociación de padres y madres del alumnado (AMPA).

La modalidad de Colaboración con el entorno (Collaborating with community) hace referencia a las relaciones que el centro educativo establece con el contexto sociocultural próximo, como mecanismo para complementar la formación de la escuela. El cometido de las familias en este caso será el de apoyar este tipo de prácticas.

La inclusión de estos dos modelos, Epstein (2001) y Vogels, (2002), aportan una base teórica tanto para el diseño de la recogida de datos, como para la sistematización de los resultados obtenidos. La adaptación de estos modelos a la investigación sobre participación es propia y original de este estudio, ya que no hemos encontrado estudios empíricos previos que incorporen como marco teórico estos referentes.

\section{PROPÓSITOY OBJETIVOS}

El propósito general que mueve a esta investigación es el de explorar la participación de las familias en los centros de educación primaria, desde una doble vertiente; tipo de oferta que realizan las escuelas y respuesta familiar a la misma. Los modelos de participación identificados, el de Epstein que conceptualiza los tipos de oferta de participación y el de Vogels, que teoriza sobre los modelos de respuesta de las familias, sirvieron para fundamentar los objetivos científicos de esta investigación que se concretan en:

a) Conocer la respuesta de las familias a la oferta de cooperación que hacen los centros educativos.

b) Identificar motivaciones y concepciones que llevan a los padres de alumnos a su participación en el centro de educación primaria.

\section{METODOLOGÍA}

Esta investigación utiliza un diseño descriptivo, tipo encuesta, con un enfoque metodológico cuantitativo.

La población objeto de estudio, según datos facilitados por la Consejería de Educación de la Junta de Andalucía, la conforman un total de 41.799 padres/madres del alumnado matriculado en la Educación Primaria en Sevilla, en el curso 2011/2012. La muestra, extraída con un nivel de confianza del 95\% y un error muestral del 10, asciende a un total de 110 familias participantes.

El tipo de muestreo utilizado fue, en primer lugar, estratificado y por conglomerados (centros educativos) en el que se tuvieron en cuenta los once distritos en los que se divide la ciudad, sin excluir ninguno de ellos para que la muestra fuera lo más representativa posible. Posteriormente, en cada distrito se realizó un muestreo estratificado, atendiendo a la tipología de los centros y teniendo en cuenta que en Sevilla existen 161 colegios, 93 públicos (58\%) y 68 concertados (42\%). Por lo tanto se seleccionó un centro privado y otro público en cada distrito para respetar dicha proporción. Finalmente, en 
cada división entre centros públicos y privados se realizó un muestreo aleatorio simple que nos indicó los centros públicos y privados que serían objeto de estudio en cada uno de los once distritos de la ciudad. Dando como resultado un total de 22 centros participantes, siendo la mitad centros docentes privados y la otra mitad públicos.

El $22,7 \%$ de los cuestionarios fueron contestados por padres, el $75,5 \%$ por madres y sólo un 1,8\% por ambos. La estructura familiar es nuclear en un $88,2 \%$ de los casos y monoparental en un $11,8 \%$.

En la figura 1 se muestran otros datos sociológicos de la muestra de este estudio.

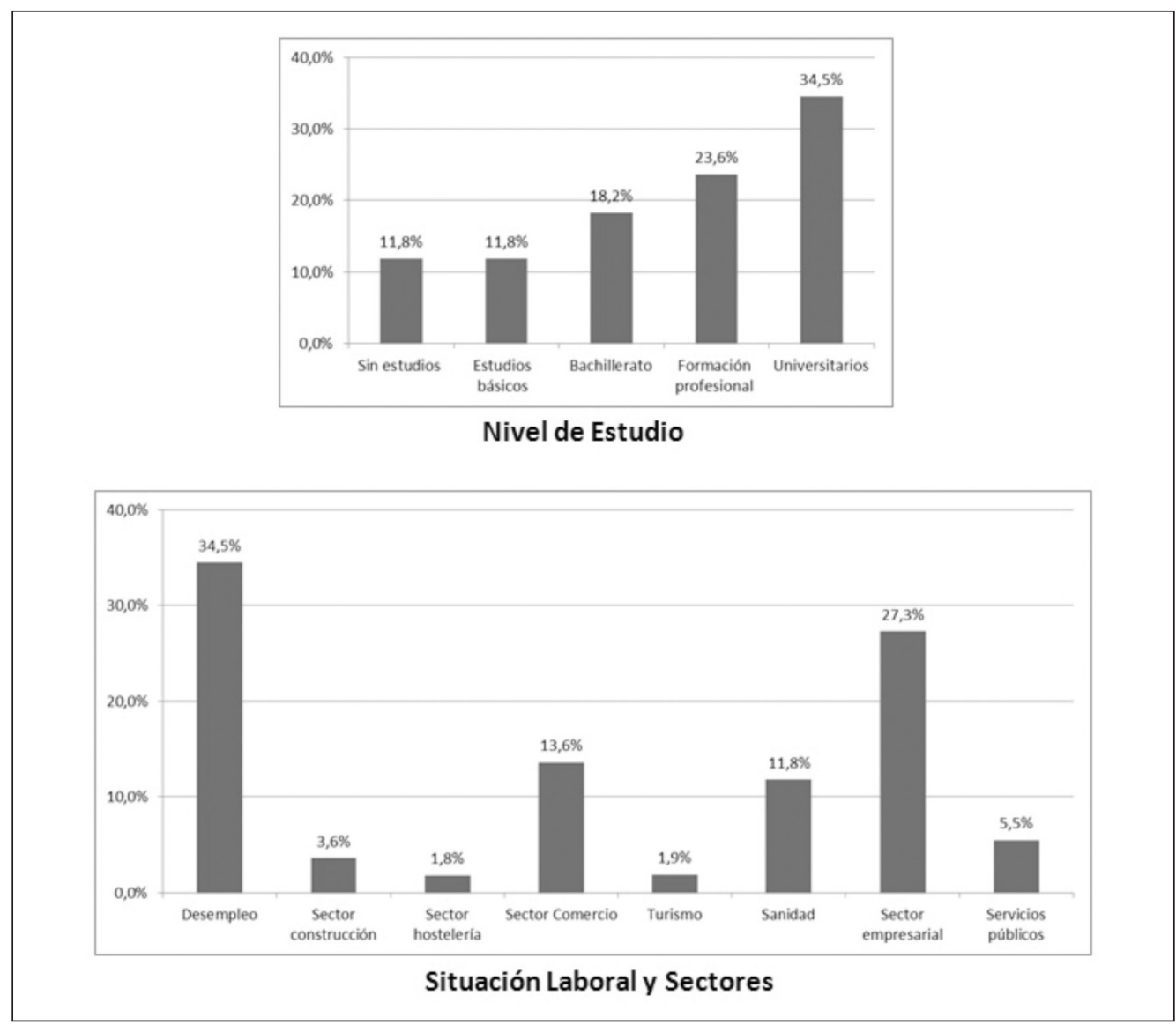

FIGURA 1

DATOS SOCIOLÓGICOS DE LA MUESTRA

La información de la figura 1 nos indica que la muestra es muy heterogénea, tanto en el nivel de estudios como en su situación profesional destacando, que más del $70 \%$ de la muestra tiene estudios superiores a la formación básica. Y en cuanto a la situación profesional, queda muy palpable el elevado índice de desempleo, reflejo de la situación económica del momento. 
Para la recogida de los datos se utiliza un cuestionario construído ad hoc para responder de forma precisa a los objetivos científicos planteados. Los modelos de participación de Vogels (2002) y Epstein (2001) sirven de referencia para su elaboración. De ahí que se incluyan cuestiones referidas al modelo que propone Epstein (2001) en las seis modalidades: ayuda a los padres (parenting), comunicación (communicating), voluntariado (volunteering), aprendizaje en casa (learning at home), toma de decisiones (decision making), y colaboración con el entorno (collaborating with community).

Así, los doce primeros ítems hacen referencia a los tipos de participación que los centros educativos proponen a los padres y que se corresponden con los seis tipos de Epstein ya citados. La traducción de estos a la posible oferta de los centros educativos españoles puede verse en la Tabla 1. Las respuestas a los ítems del cuestionario se registraron en una escala ordinal tipo Likert, con cinco opciones de respuesta.

TABLA 1

TIPO DE OFERTA DE PARTICIPACIÓN QUE LOS CENTROS EDUCATIVOS PLANTEAN A LAS FAMILIAS

\begin{tabular}{|c|c|c|}
\hline \multirow{7}{*}{ 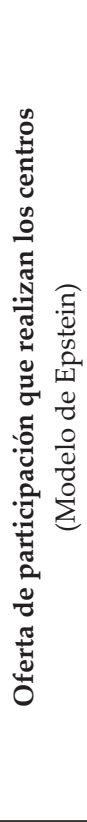 } & \multicolumn{2}{|c|}{$\begin{array}{c}\text { Acciones y actividades ofertadas por los centros educativos contempladas en la elaboración } \\
\text { del cuestionario }\end{array}$} \\
\hline & $\begin{array}{l}\text { Ayuda a los padres } \\
\text { (Parenting) }\end{array}$ & $\begin{array}{l}\text { Programas que pone en marcha el centro educativo } \\
\text { Cursos de formación que realiza la escuela }\end{array}$ \\
\hline & $\begin{array}{l}\text { Comunicación } \\
\text { (Communicating) }\end{array}$ & $\begin{array}{l}\text { Reuniones para recibir información sobre sus hijos/as } \\
\text { Contestación a la información recibida por parte del } \\
\text { colegio }\end{array}$ \\
\hline & $\begin{array}{l}\text { Voluntariado } \\
\text { (Volunteering) }\end{array}$ & $\begin{array}{l}\text { Actividades escolares } \\
\text { Actividades extraescolares }\end{array}$ \\
\hline & $\begin{array}{l}\text { Aprendizaje en casa } \\
\text { (Learning at home) }\end{array}$ & $\begin{array}{l}\text { Coordinación entre docentes y familias } \\
\text { Realización de tareas escolares en casa }\end{array}$ \\
\hline & $\begin{array}{l}\text { Toma de decisiones } \\
\text { (Decision making) }\end{array}$ & $\begin{array}{l}\text { Asociación de madres y padres del alumnado } \\
\text { Consejo Escolar del centro }\end{array}$ \\
\hline & $\begin{array}{l}\text { Colaboración con el } \\
\text { entorno } \\
\text { (Collaborating with } \\
\text { community) }\end{array}$ & $\begin{array}{l}\text { Excursiones que promueve la escuela } \\
\text { Jornadas de convivencia del centro con otros colegios }\end{array}$ \\
\hline
\end{tabular}

La propuesta de Vogels (2002) sirve de referencia para recabar información sobre la posición y respuesta de los padres respecto a la oferta de los centros educativos. En este sentido las familias, según Vogels, podrían tener distintos niveles de implicación, representados en una progresión ascendente: consumidores, clientes, participantes y socios. Los ítems elaborados de acuerdo a este modelo nos permitirían detectar qué modelo de implicación asumen las familias (no se considera el de consumidor porque está fuera de cualquier forma de cooperación). En nuestra investigación este modelo se conjuga con el anterior de Epstein (2001) dando como resultado la siguiente tabla 2. 
TABLA 2

TIPOS DE PARTICIPACIÓN Y MOTIVOS QUE HACEN EFECTIVA LA PARTICIPACIÓN DE LAS

FAMILIAS

\begin{tabular}{|c|l|l|l|}
\hline \multirow{2}{*}{$\begin{array}{c}\text { Oferta de participación } \\
\text { (Modelo de Epstein) }\end{array}$} & \multicolumn{3}{|c|}{ Motivaciones y concepciones que llevan a la participación de los padres en los } \\
centros educativos \\
(Modelo de Vogels)
\end{tabular}

Para el análisis de los datos se utilizó el software estadístico SPSS v.19. El cuestionario, en cuanto a su fiabilidad, obtiene un Alfa de Cronbach de .830. La aplicación del análisis factorial con rotación varimax arroja seis factores que explican el $69.47 \%$ de la varianza total. El primer factor expresa el 26.93\% de la varianza. Los ítems que se agrupan en torno a él son los que responden a "motivos". Por tanto, este factor identifica los motivos que llevan a las familias a participar en la escuela. El segundo factor, que explica el $13.47 \%$ de la varianza incluye los tres ítems del tipo de participación Comunicación (Communicating) y el ítem referido a la participación en las actividades escolares. El tercer factor agrupa dos ítems del tipo de participación Colaboración con el entorno (Collaborating with community), y explica el $11.10 \%$ de la varianza. El cuarto factor describe el $6.46 \%$ de la varianza y aglutina los ítems referidos a la participación en el AMPA y en el Consejo Escolar, así como en las actividades extraescolares representando la denominación de Toma de decisiones (Decisión making). En el quinto factor 
se reúnen los ítems relacionados con la modalidad Ayuda a los padres (Parenting), y explican el $5.80 \%$ de la varianza. Y por último el sexto factor, con un $5.69 \%$ de la varianza agrupa los ítems correspondientes a la denominación de Aprendizaje en casa (Learning at home), representando la responsabilidad de las familias en el aprendizaje. Por tanto estos resultados nos muestran la validez de contenido en cuanto a identificación y representación de las dimensiones del modelo de referencia adoptado.

\section{RESULTADOS}

\section{I. Respuesta de las familias a la oferta de participación que proponen los centros educativos}

La respuesta de las familias a la oferta de participación que ofrecen los centros educativos queda sintetizada en la figura 2.

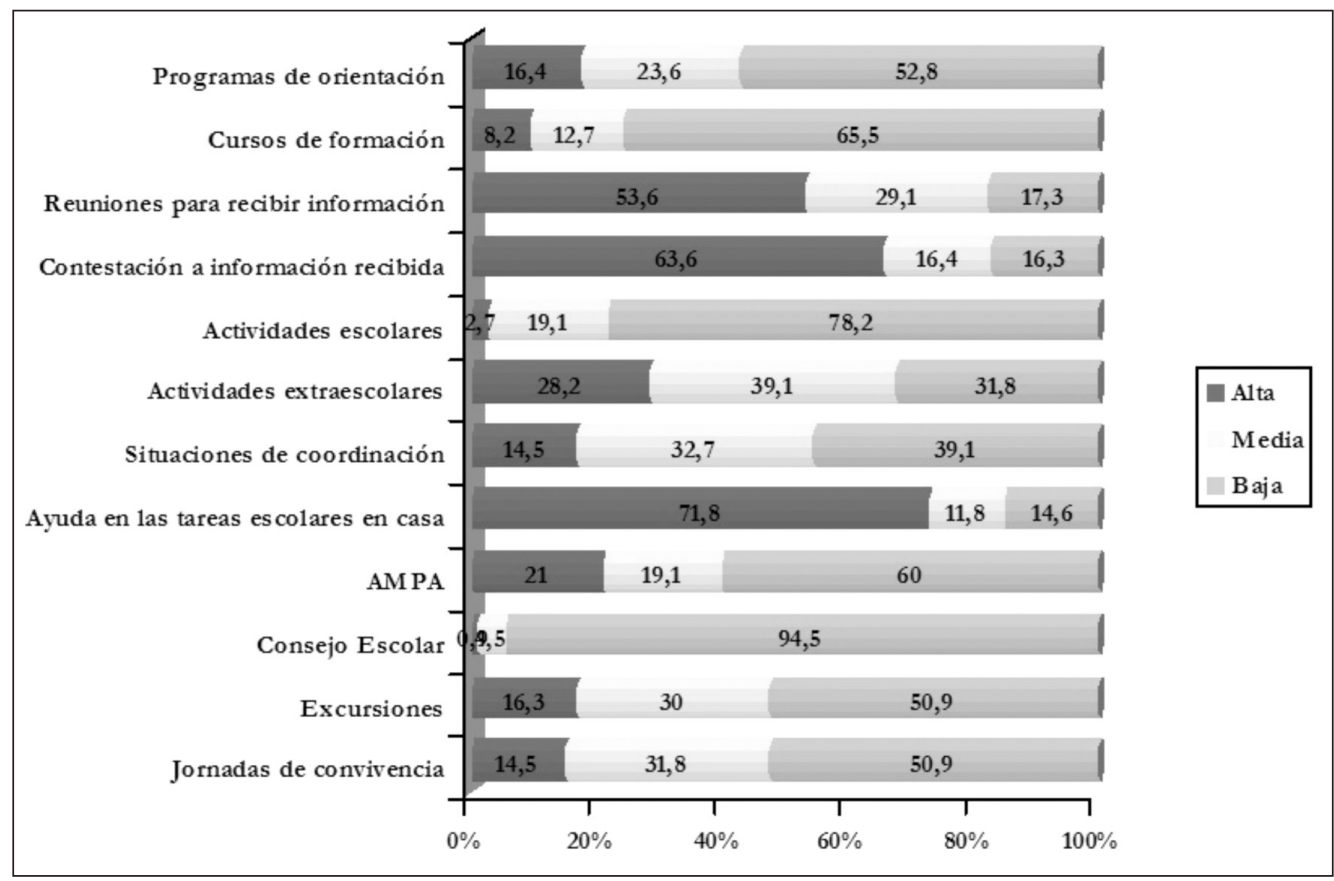

\section{FIGURA 2}

\section{RESULTADOS DE LA RESPUESTA DE LAS FAMILIAS A LA OFERTA QUE LOS CENTROS} REALIZAN, EXPRESADO EN PORCENTAJES

La gráfica muestra como datos a destacar, la baja participación de las familias en el Consejo Escolar del centro, ya que un $94.5 \%$ aseguran no haber participado nunca o muy poco. De igual manera es destacable la baja implicación en las actividades escolares que se realizan en el centro, un $78.2 \%$ de las respuestas señalan no haber participado nunca o hacerlo de forma muy discreta. 
Sin embargo, esos bajos niveles de participación, chocan con los altos porcentajes de implicación que se obtienen en la ayuda en las tareas escolares a realizar en casa. El 71.8\% de padres/madres aseguran participar siempre o bastante en este cometido.

Una interpretación de los resultados obtenidos, desde el modelo teórico de Epstein, nos lleva a indicar que las propuestas de participación definidas como Toma de decisiones (Decision making), representadas por la participación en el AMPA o en el Consejo Escolar, son las que menos acogida tienen por parte de las familias encuestadas. De igual forma, las actividades referidas a Ayuda a los padres (Parenting) ocupan el segundo puesto en el podio de la baja participación, ya que los dos ítems que se relacionan con este tipo muestran unos porcentajes medio-altos. Completaría ese podio, la Colaboración con el entorno (Collaborating with community), en que los ítems que corresponden con esta modalidad, tales como "Participación en excursiones" y "Participación en jornadas de convivencia con otros centros", obtienen en torno a un $50 \%$ de nula o baja participación.

Por el contrario, el tipo Comunicación (Communicating) es el de mayor receptividad en las familias, ya que los ítems "Participar en reuniones para recibir información" y "Contestación a la información recibida" obtienen una intensidad alta en torno al 50\% y al 65\% de las respuestas. En síntesis, los resultados expresados en la gráfica 1 muestran niveles diferenciados de implicación de las familias según las propuestas de participación. Así, se identifica alta participación en las actividades incluidas en las modalidades de Comunicación y Aprendizaje en casa (71,8\%). Mientras se observa baja participación en las modalidades de Toma de decisiones, Ayuda a los padres, y Colaboración con la comunidad. Estos resultados muestran un perfil cultural que marca la relación de los padres con la escuela, indicando que asumen la educación más desde una responsabilidad individual que colaborativa y/o participativa.

\subsection{Motivos que Ilevan a las familias a participar en el centro educativo}

El modelo teórico de Vogels en el que se identifican tres modalidades de concepciones: clientes, participantes y socios, y por tanto, de motivaciones, es utilizado tanto para recabar información, como para sistematizar los resultados obtenidos. La proyección de estos modelos en ítems de respuesta se concretan en tres opciones: a) porque lo recomiendan los docentes (clientes), b) porque así puedo proponer ideas (participantes) y c) porque es fundamental para el éxito académico y personal de mi hijo/a (socios). Los resultados que se muestran en la figura 3, indican que la motivación se orienta preferentemente hacia el modelo de clientes, alcanzando los porcentajes más elevados en todas las opciones de participación. Existe una excepción en esta tendencia y es la de coordinación y tareas escolares en que se empata con la motivación de "porque así puedo proponer ideas". También como segunda excepción encontramos la participación en programas y cursos, aspecto que les motiva a la valoración de sentirse una parte fundamental para el éxito académico y personal de su hijo/a. Estos resultados nos indican que se conjugan varios modelos de participación, con un ligero predominio del modelo clientes. Por tanto nos posibilita aventurar la idea de que las familias tienen una posición activa en la educación de sus hijos en el sistema escolar. Resulta 
esperanzador observar los elevados porcentajes de respuesta en el modelo más activo correspondiente al de socios.

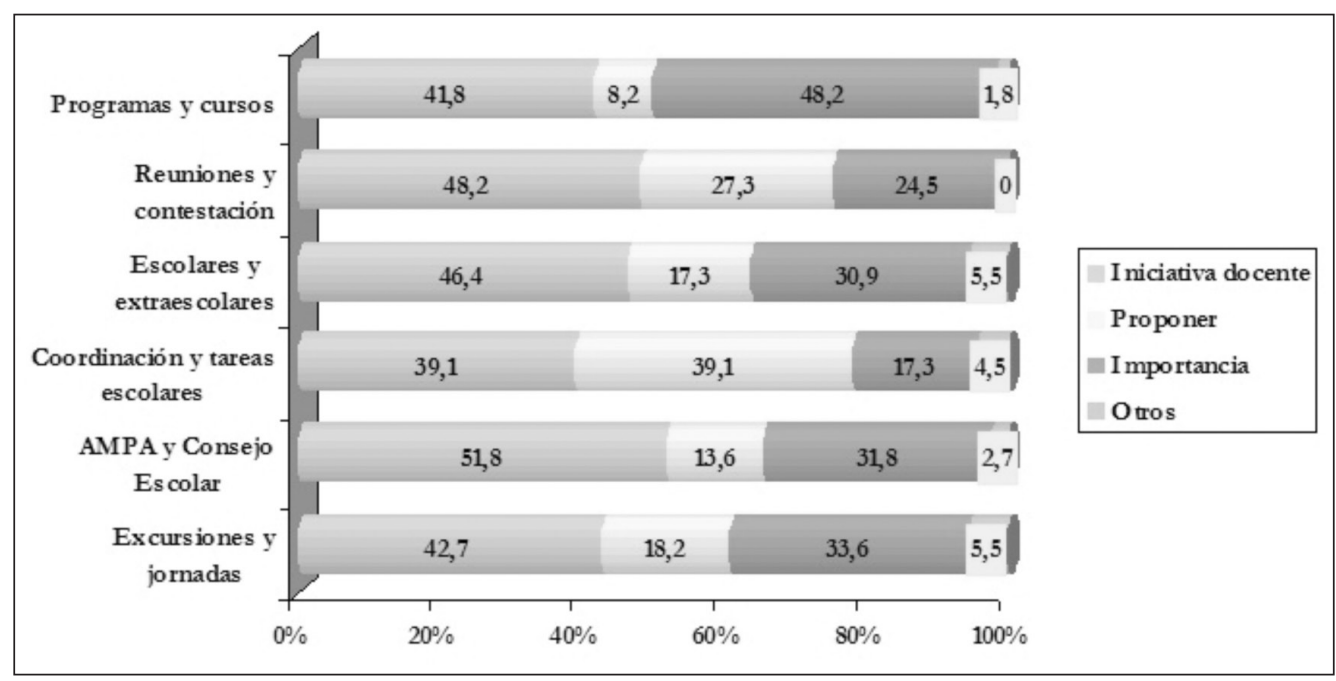

FIGURA 3

RESULTADOS SOBRE MOTIVOS QUE LLEVAN A LAS FAMILIAS A PARTICIPAR, EXPRESADO EN PORCENTAJES

Como síntesis, la gráfica nos muestra que la principal motivación, en los seis tipos de participación excepto el primero, es la iniciativa docente. Sin embargo si sumamos, las respuestas en las opciones correspondientes a participantes o socios superan las respuestas de la opción cliente. Por tanto estos resultados pueden leerse como la coexistencia de visiones y posicionamientos que conllevan tres niveles distintos de compromiso con la educación.

\section{CONCLUSIONESY PROPUESTAS DE MEJORA}

La participación de las familias en la escuela es un indicador relevante de la calidad del Sistema Educativo. De ahí que conocer las respuestas que las familias dan a la oferta de participación que realizan los centros educativos sea importante, en tanto permite detectar y visualizar posibles sinergias entre los principales agentes educativos, familia y escuela. En este sentido los resultados obtenidos nos permiten concluir que, por lo general, la participación de las familias en los centros educativos es baja en determinadas ofertas de participación, y positiva y/o activa en otras.

Las actividades que tienen una respuesta más baja por parte de las familias son la Colaboración en actividades escolares, con un $48.2 \%$ de personas que respondieron no haber participado nunca, o la pertenencia al Consejo Escolar, donde los porcentajes se sitúan en un $74.5 \%$ de respuestas que afirman no participar nunca en tal órgano. Estos resultados son similares a los hallados por Lozano, Alcaraz y Colás, (2014) en la 
comunidad murciana, Otros estudios anteriores, (Martín-Moreno 2000 o Garreta 2008), también arrojan bajos niveles de participación de las familias en las AMPA y en los Consejos Escolares. Es decir la modalidad Toma de decisiones parece tener poca aceptación por parte de las familias, así como la Ayuda a los padres y la Colaboración con el entorno.

Sin embargo las familias parecen implicarse más en las tareas escolares que se proponen para hacer en casa $(71.8 \%$ de alta participación). Por tanto, la modalidad Aprendizaje en casa (Learning at home), es la preferente de las familias, así como la Comunicación (Communicating). En este sentido podemos concluir que estas dos últimas modalidades de oferta de colaboración, son las que reciben mejor aceptación por parte de los padres. No obstante la heterogeneidad de respuestas nos lleva a pensar que podrían investigarse perfiles de respuestas, obteniendo tipologías de participación. En este sentido otras investigaciones (Lozano, Alcaraz y Colás, 2013) han detectado dos perfiles de comportamiento diferenciados en cuanto a su participación en los centros educativos. Otra línea de indagación que se abre es relacionar la participación con otras variables tales como concepciones de la educación, variables socioculturales, niveles de formación, etc.

Una valoración global de los resultados sobre los motivos que llevan a las familias a participar, nos hace concluir que coexisten tres posicionamientos. Si bien la opción de "clientes" arroja porcentajes altos en todas las propuestas de participación, (entre un $39,1 \%$ al 51,8\%) también tienen presencia las motivaciones: "participantes" (entre 8,2\% y $39,1 \%)$ y la de "socios" (17,3\% y 48,2\% ), con unos índices menores que la de "cliente", pero que resultan prometedores por el significado que tiene de transformación hacia un mayor compromiso de los padres en los centros educativos.

Por tanto, la prevalencia del modelo cliente, unido a los altos índices de participación familiar en lo relacionado con las tareas escolares para casa, nos lleva a pensar que la mayoría de las familias encuestadas tienen una concepción de la educación en la que cada personaje tiene su espacio delimitado. Así, al profesorado le corresponderá el centro educativo, y a las familias el hogar. No obstante la coexistencia de distintos niveles de implicación, abre perspectivas optimistas en cuanto a la posible evolución de las respuestas actuales.

Para finalizar, y como propuesta de mejora general en el tema que nos ocupa estimamos que estos resultados pueden servir de reflexión a los responsables de los centros educativos, cara a arbitrar estrategias que potencien una mayor participación y compromiso de las familias con la tarea educativa. En este sentido la formación, a través de la escuela de padres, puede ser un elemento impulsor de la participación. Por otro lado, la identificación de heterogeneidad de posicionamientos en cuanto a motivaciones de las familias para participar, es otro aspecto relevante a considerar por las organizaciones, en tanto estas pueden trabajar para conseguir mayores niveles de implicación y compromiso de las familias, a través de medidas orientadas a la creación de un ambiente y cultura de participación. Por tanto, y como síntesis las propuestas de mejora se podrían articular sobre dos ejes; la formación y la transformación de la cultura de los centros educativos, cimentadas a su vez en el análisis, la reflexión e investigación de lo que acontece en los contextos educativos reales. Este es un camino irrenunciable, si se quiere apostar por la calidad de las instituciones educativas, el cumplimiento de sus funciones sociales y la excelencia de los resultados escolares. 


\section{BIBLIOGRAFÍA}

Barton, C. (2001). Underprivileged Urban Mothers' Perspectives on Science. Journal of Research in Science Teaching, 38, 688-711.

Chiu, M. y Xihua, Z. (2008). Family and motivation effects on mathematics achievement: Analyses of students in 41 countries. Learning and Instruction, 18, 321-336.

Comisión Europea (2000). Informe Europeo sobre la Calidad de la Educación Escolar.

Consejo Escolar del Estado (2011). Informe sobre el estado y situación del sistema educativo. Madrid. España.

Córdoba, L., García, V., Luengo, L., Vizuete, M. y Feu, S. (2011). Determinantes socioculturales: su relación con el rendimiento académico en alumnos de Enseñanza Secundaria Obligatoria. Revista de Investigación Educativa, 29(1), 83-96.

Dearing, E., Kreider, S. y Weiss, H. (2006). Family involvement in school and lowincome children's literacy performance: Longitudinal associations between and within families. Journal of Educational Psychology, 98, 653-664.

Driessen, G., Smit, F. y Sleegers, P. (2005). Parental Involvement and Educational Achievement. British Educational Research Journal, 31, 509-532.

Epstein, J. L. (2001). School, family, and community partnerships: preparing educators and improving schools. Boulder, Colorado. Westview Press.

Epstein, J. L., and al. (2002). School, family and community partnerships: Your handbook for action. Thousand Oaks, CA. Corwin Press.

Fan, W., Williams, C. y Wolters, C. (2011). Parental Involvement in Predicting School Motivation: Similar and Differential Effects Across Ethnic Groups. The Journal of Educational Research, 105, 21-35.

Fraguela, R., Lorenzo, J. y Varela, L. (2011). Escuela, familias y ocio en la conciliación de los tiempos cotidianos de la infancia. Revista de Investigación Educativa, 29(2), 429-446.

Garreta, J. (2008). La participación de las familias en la escuela pública. Madrid: CEAPA.

Jiménez, C. (2006). Educación familiar y alumnos con alto rendimiento. Revista Española de Pedagogía, 64(234), 273-302.

Ley Orgánica 2/2006, de 3 de mayo, de Educación (BOE número 106 de 4/5/2006).

Lozano, J. Alcaraz, S. y Colás, P. (2013). Los centros educativos multiculturales y sus relaciones con las familias: el caso de la región de Murcia. Revista Educación XX1. 16.1, pp. 210-232.

Lozano, J. Alcaraz, S. y Colás, P. (2014). La escuela multicultural. Como la valoran y participan las familias de la Region de Murcia. Scientific Journal On Intercultrual Studies. En proceso de revisión.

Martín-Moreno, Q. (2000). Bancos de talento: Participación de la comunidad en los centros docentes. Madrid: Sanz y Torres.

OECD (2012). Let's Read Them a Story! The Parent Factor in Education, PISA, OECD Publishing.

Pomerantz, E., Moorman, E. y Litwack, S. (2007). The how, whom, and why of parents' involvement in children's academic lives: More is not always better. Review of Educational Research, 77(3), 373-410.

Ruiz de Miguel, C. (2009). Las escuelas eficaces: Un estudio multinivel de factores explicativos del rendimiento escolar en el área de matemáticas. Revista De Educación, 348, 355-376. 
Sanders, M. (2005). Building School-Community Partnerships: Collaboration for Student Success. Harvard Educational Review, 75, 133-173.

Santos Guerra, M. (1997). El crisol de la participación: Estudio etnográfico sobre la participación en consejos escolares de centro. Madrid: Escuela española.

Santos, M. A., Lorenzo M. y Priegue, D. (2011). Infancia de la inmigración y educación: la visión de las familias. Revista de Investigación Educativa, 29(1), 97-110.

Sarramona, J., y Roca, E. (2007). La participación de las familias en la escuela como factor de calidad educativa. Participación Educativa, 4, 25-33.

Villiger, C., Niggli, A. y Wandeler, C. (2011). Does family make a difference? Mid-term effects of a school home based intervention program to enhance reading motivation. Learning and Instruction, 22, 79-91.

Vogels, R. (2002). Ouders bij de Les. Betrokkenheid van Ouders bij de School van hun Kind. Den Haag, Sociaal Cultureel Planbureau.

Fecha de recepción: 3 de enero de 2013.

Fecha de revisión: 3 de enero de 2013.

Fecha de aceptación: 24 de abril de 2013. 
